\title{
Performance of administrative case definitions for comorbidity in multiple sclerosis in Manitoba and Nova Scotia
}

\author{
R. A. Marrie, MD, PhD (1, 2); J. D. Fisk, PhD (3, 4, 5); K. J. Stadnyk, MSc (5); H. Tremlett, PhD (6); \\ C. Wolfson, PhD (7, 8); S. Warren, PhD (9); V. Bhan, MD (3, 5); B. N. Yu, MD, PhD (1, 2), for the CIHR Team in the \\ Epidemiology and Impact of Comorbidity on Multiple Sclerosis
}

This article has been peer reviewed.

\begin{abstract}
Introduction: As the population ages and the prevalence of comorbid conditions increases, the need for feasible, validated methods of comorbidity surveillance in chronic diseases such as multiple sclerosis (MS) increases.

Methods: Using kappa (к) statistics, we evaluated the performance of administrative case definitions for comorbidities commonly observed in MS by comparing agreement between Manitoba (MB) administrative data and self-report $(\mathrm{n}=606)$ and Nova Scotia (NS) administrative data and self-report $(\mathrm{n}=1923)$.
\end{abstract}

Results: Agreement between the administrative definitions and self-report was substantial for hypertension $(\kappa=0.69$ [NS], $0.76[\mathrm{MB}])$ and diabetes $(\kappa=0.70$ [NS], $0.66[\mathrm{MB}])$; moderate for hyperlipidemia $(\kappa=0.53[\mathrm{NS}], 0.51[\mathrm{MB}])$ and heart disease $(\kappa=0.42[\mathrm{NS}], 0.51[\mathrm{MB}])$ and fair for anxiety $(\kappa=0.27[\mathrm{NS}], 0.26[\mathrm{MB}])$. In NS, agreement was substantial for inflammatory bowel disease $(\kappa=0.71)$ and moderate for epilepsy $(\kappa=0.48)$.

Conclusion: Administrative definitions for commonly observed comorbidities in MS performed well in 2 distinct jurisdictions. This suggests that they could be used more broadly across Canada and in national studies.

Keywords: administrative data, validation, comorbidity, multiple sclerosis

\section{Introduction}

Comorbidities in many chronic diseases are associated with a broad range of adverse outcomes including increased disability, mortality and health care utilization. ${ }^{1}$ For example, vascular comorbidities such as hypertension are associated with more rapid cognitive decline in people with Alzheimer disease. ${ }^{2}$ Multiple sclerosis (MS) is a chronic neurological disease affecting more than 2 million people worldwide. $^{3}$ It has a high incidence and prevalence in Canada, ${ }^{4}$ with annual incidence rates as high as 23.9 per 100000 population $^{5}$ and an estimated crude prevalence of 240 per 100000 population in 2000/2001. ${ }^{6}$ Physical and mental comorbidities are common in people with MS. Comorbidities have been associated with diagnostic delays, greater disability and lower quality of life. ${ }^{7-10}$ However, relatively few methods exist to enable the valid and feasible measurement of comorbidities in various settings in MS, ${ }^{11}$ despite the need to better understand the impact of comorbidities on MS. This is particularly true at the population level, where methods are needed to assess the impact of MS comorbidities on health services, hospitalizations, physician visits, preventive care and access and other population-level outcomes such as mortality. ${ }^{12}$

Potential data sources to identify the presence of comorbidities at the population level include medical records reviews,

\section{Author references:}

1. Department of Internal Medicine, University of Manitoba, Winnipeg, Manitoba, Canada

2. Department of Community Health Sciences, University of Manitoba, Winnipeg, Manitoba, Canada

3. Department of Medicine, Dalhousie University, Halifax, Nova Scotia, Canada

4. Department of Psychiatry, Dalhousie University, Halifax, Nova Scotia, Canada

5. Capital District Health Authority, Halifax, Canada

6. Department of Medicine (Neurology), University of British Columbia, Vancouver, British Columbia, Canada

7. Department of Epidemiology and Biostatistics and Occupational Health, McGill University, Montréal, Quebec, Canada

8. Research Institute of the McGill University Health Centre, Montréal, Quebec, Canada

9. Faculty of Rehabilitation Medicine, University of Alberta, Edmonton, Alberta, Canada

CIHR Team in the Epidemiology and Impact of Comorbidity on Multiple Sclerosis includes Ruth Ann Marrie (University of Manitoba, Winnipeg, Manitoba), Bo Nancy Yu (University of

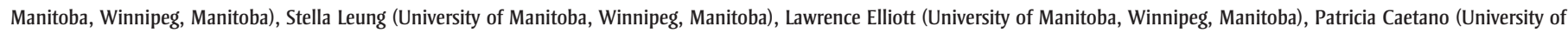
Manitoba, Winnipeg, Manitoba), James F. Blanchard (University of Manitoba, Winnipeg, Manitoba), Lawrence W. Svenson (University of Alberta, Edmonton, Alberta), Joanne ProfettoMcGrath (University of Alberta, Edmonton, Alberta), Sharon Warren (University of Alberta, Edmonton, Alberta), Christina Wolfson (McGill University, Montréal, Quebec), Nathalie Jette (University of Calgary, Calgary, Alberta), Scott B. Patten (University of Calgary, Calgary, Alberta), Charity Evans (University of Saskatchewan, Saskatoon, Saskatchewan), Helen Tremlett

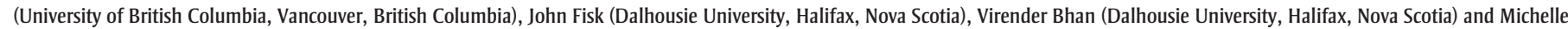
Ploughman (Memorial University, St. John's, Newfoundland).

Correspondence: Ruth Ann Marrie, Departments of Internal Medicine and Community Health Sciences, University of Manitoba, GF-533 Health Sciences Centre, 820 Sherbrook Street, Winnipeg, MB R3A 1R9; Tel.: 204-787-4951; Fax: 204-787-1486; Email: rmarrie@hsc.mb.ca 
surveys (interviews or self-report) and administrative (health claims) data. ${ }^{13}$ No single source is ideal for every study design-or even for every comorbidity. ${ }^{14}$ In some countries, including Canada, administrative data offer an attractive opportunity for cost-effective, populationbased comorbidity research ${ }^{15}$ and have a track record of successful use for national surveillance of chronic diseases such as diabetes. ${ }^{16}$ Administrative case definitions for several physical and mental comorbidities among people with MS have been validated in one Canadian province, Manitoba. ${ }^{17-20}$ However, the characteristics of physician claims databases and billing practices vary across jurisdictions: ${ }^{21}$ the number of diagnosis fields permitted in physician claims varies, as does the specificity of diagnosis codes based on the number of digits coded, both of which may affect the sensitivity and specificity of case definitions. Thus, the need to assess the performance of such methods in other jurisdictions remains and is particularly important if findings using these methods are to be compared across jurisdictions.

In this study, we compared the performance characteristics of previously developed administrative case definitions for comorbidity in the MS population of 2 Canadian provinces, Manitoba and Nova Scotia, with the goal of establishing methods that could be used to study comorbidity in the MS population across Canada.

\section{Methods}

This validation study involved the secondary analysis of linked clinical and administrative data in 2 provinces, Manitoba and Nova Scotia. We present the methods for the work in Manitoba followed by the methods for the work in Nova Scotia.

\section{Manitoba}

\section{Setting}

The province of Manitoba has a stable population of nearly 1.2 million. ${ }^{22}$ The data sources used included provincial health claims data for the period 2001 to 2006 and self-report data from 604 patients with MS covering their lifetime through 2006. The University of Manitoba's Research Ethics Board gave ethical approval for this portion of the study. The Manitoba Health Information Privacy Committee approved administrative data access.

\section{Administrative data}

Manitoba Health is the provincial government department responsible for health care delivery to $98 \%$ of the Manitoba population. ${ }^{22}$ Manitoba Health maintains computerized records of all submitted health services claims, including hospital and physician claims. These claims include a unique personal health identification number (PHIN) for the resident who received the service. The population registry captures sex, dates of birth and death, and dates of provincial health insurance coverage.
Hospital discharge abstracts include the unique PHIN, dates of admission and discharge, and up to 16 diagnostic codes. Before 2004 these diagnoses were recorded using 5-digit International Classification of Disease (ICD)-9 codes, and since 2004 the diagnoses have been recorded using ICD-10-CA codes. We used all the diagnoses reported in the discharge abstract database. Each physician claim includes the unique PHIN and 3-digit ICD-9 code for one physician-assigned diagnosis. To protect confidentiality, the linkage in this study (see below) was performed via scrambled PHIN, using anonymized versions of the administrative databases provided by Manitoba Health.

\section{Administrative case definitions of comorbidity}

The case definitions used in this study were previously developed and validated in Manitoba using a cohort of people with MS (see Table 1). ${ }^{17-19,23}$ Briefly, we generated lists of ICD-9/10 codes that matched the clinical terms for the comorbidities of interest, with these comorbidities chosen based on their perceived importance for MS care. Specifically, these comorbidities were either reported to affect more than $5 \%$ of MS patients or the literature suggested an association with clinical outcomes. ${ }^{7-10,24}$ We developed several case definitions for each condition, varying the number of hospital and physician claims required and the number of years of data used to classify a person as being affected. ${ }^{25}$

TABLE 1

Administrative (health claims) case definitions used for identifying comorbidity in people with multiple sclerosis in Nova Scotia and Manitoba, Canada

\begin{tabular}{|c|c|c|c|c|}
\hline Comorbidity & ICD-9 codes & ICD-10 codes & Number of years of data & $\begin{array}{l}\text { Number and type of hospi- } \\
\text { tal or physician claims }\end{array}$ \\
\hline Hypertension & $401-405$ & $\mathrm{I10-I13,} \mathrm{I15}$ & 2 & $\geq 1 \mathrm{H}$ or $\geq 2 \mathrm{P}$ \\
\hline Hyperlipidemia & 272 & E780, E782, E784, E785 & 5 & $\geq 1 \mathrm{H}$ or $\geq 2 \mathrm{P}$ \\
\hline Diabetes & 250 & E10-E14 & 5 & $\geq 1 \mathrm{H}$ or $\geq 2 \mathrm{P}$ \\
\hline Heart Disease & $410-414$ & $120-125$ & 5 & $\geq 1 \mathrm{H}$ or $\geq 2 \mathrm{P}$ \\
\hline Inflammatory bowel disease & 555,556 & K50, К51 & & $\begin{array}{l}\geq 5 \mathrm{H} \text { or } \mathrm{P} \text { or, if resident in } \\
\text { province }<2 \text { years: } \geq 3 \mathrm{H} \text { or } \mathrm{P}\end{array}$ \\
\hline Epilepsy & 345 & G40, G41 & 3 & $\geq 1 \mathrm{H}$ or $\geq 2 \mathrm{P}$ \\
\hline Depression & 296.2, 296.3, 298.0, 300.4, 311 & F32-F34 & 2 & $\geq 1 \mathrm{H}$ or $\geq 4 \mathrm{P}$ \\
\hline Anxiety & $300.0,300.2$ & F40, F41 & 2 & $\geq 1 \mathrm{H}$ or $\geq 3 \mathrm{P}$ \\
\hline
\end{tabular}

Abbreviations: ICD, International classification of diseases; $\mathrm{H}$, hospital claims; P, physician claims.

${ }^{\text {a }}$ For physician claims, the ICD-9 codes were truncated after the $3^{\text {rd }}$ digit. 


\section{Manitoba validation cohort}

We compared classification of comorbidity according to the administrative case definitions to comorbidity diagnoses based on a reference standard in 604 people with demyelinating disease, aiming to optimize agreement as measured by the kappa statistic $(\kappa)$ between the 2 data sources. These 604 people were drawn from 2 studies; ${ }^{26,27}$ they self-reported their comorbidities using a questionnaire, ${ }^{27}$ agreed to medical records review and consented to linkage of their administrative and clinical data. In each of the 2 studies, a trained abstractor blinded to the administrative data used the same standardized data collection form to abstract comorbidity data from the medical record. Participants in one study were asked about a longer list of comorbidities than in the other study, but the formatting of the questions was consistent across both studies. Findings on the validity of these administrative case definitions compared to medical records review have been published elsewhere. ${ }^{17-20}$

For this study, we selected for further evaluation case definitions with reasonable performance characteristics ${ }^{17-19,23,28}$ based on sensitivity, specificity and kappa when compared to medical records. Table 1 shows the ICD-9/10 codes and the combination of hospital and physician claims and number of years of data required to meet the various case definitions. The sensitivity and specificity of self-report and medical records for comorbidity may differ, and using different reference standards may contribute unnecessary heterogeneity when comparing the validity of administrative case definitions across studies. ${ }^{29}$ We therefore compared the performance of the administrative case definitions to self-report to facilitate comparisons to Nova Scotia where the reference standard was self-report.

People meeting the case definitions were considered affected from the date of the first relevant health claim for these conditions.

\section{Nova Scotia}

\section{Setting}

The eastern province of Nova Scotia has a population of approximately $945000 .^{30}$ The data sources for the study included provincial health claims (administrative) data for Nova Scotia held by the Population Health Research Unit at Dalhousie University for the 20-year period from 1990 to 2010, and self-report data from patients attending the Dalhousie Multiple Sclerosis Research Unit (DMSRU). Nova Scotia's Capital Health Research Ethics Board gave ethical approval for this portion of the study.

\section{Administrative data}

Nova Scotia's provincial health insurance program provides publicly funded care to all residents and maintains computerized records of all submitted health service claims including hospital, physician and prescription claims. Each resident has a unique health care identification number attached to each health service claim. The Insured Patient Registry captures the sex, dates of birth and dates of death for each beneficiary of health services as well as the dates of provincial health insurance coverage.

Hospital discharge abstracts include the patient's health care identification number and diagnostic codes reported using 5digit ICD-9 codes (up to 2001) or ICD-10CA codes (as of 2001). We used all available diagnoses reported in the discharge abstract database. From 1989 through 1991 up to 5 diagnoses could be reported, from 1992 through 1995 up to 7 could be reported and from 1996 up to 16 could be reported.

Physician claims include the patient's health care identification number, date of service and an ICD-9 code for the diagnosis. Before 1997 the ICD-9 codes used only 3 digits; after 1997 up to 5 digits could be submitted although 3-digit codes are most commonly used. ${ }^{21}$ We used 3digit ICD-9 codes by truncating the last 2 digits (Table 1) for physician claims to ensure (1) comparability with the administrative case definitions developed in Manitoba; (2) consistency over time in Nova Scotia; and (3) generalizability to other jurisdictions, including Saskatchewan, Ontario, Prince Edward Island and Newfoundland and Labrador, that also use 3-digit codes. ${ }^{21}$ This approach is also consistent with that used by the Canadian Chronic Disease Surveillance System (CCDSS, formerly known as the
National Diabetes Surveillance System). The CCDSS has developed case definitions for chronic diseases such as diabetes that rely on the "lowest common denominator" to ensure case definitions can be applied in all jurisdictions. ${ }^{16}$ Between 1989 and 1996, Nova Scotia physicians submitted only one diagnosis per claim, but as of 1997 they could submit up to 3 . Again, to ensure comparability and generalizability of these definitions across Canada and over time, we used only the primary (first) diagnosis submitted for each physician claim in our primary analysis. As a sensitivity analysis, we also evaluated the impact of using all 3 diagnoses submitted on the physician claims expecting that this would improve the sensitivity of administrative data.

\section{Nova Scotia study population}

We had previously developed and tested several administrative case definitions for MS, comparing these to medical records including clinic letters, MRI reports, cerebrospinal fluid results and evoked potentials reports. ${ }^{26}$ We subsequently evaluated the performance of these case definitions in Nova Scotia. ${ }^{31}$ Based on this work, we applied a case definition of 3 or more hospital (any diagnosis field) or physician claims (the primary diagnosis only) for MS. To this population we applied the case definitions for comorbidity developed in Manitoba, the sole exception being the definition for inflammatory bowel disease. Since small numbers of reported cases had precluded validation of this case definition in the MS population in Manitoba, we instead used a case definition that has been validated in the general populations of Manitoba and Quebec. ${ }^{32,33}$

\section{Dalhousie Multiple Sclerosis Research Unit clinical database}

The DMSRU, located within a tertiary care hospital, is the only provider of MS specialty care in Nova Scotia. The DMSRU has systematically documented each patient visit since 1980. Since 1998, all Nova Scotia residents who wish to receive provincial funding for MS-specific (disease-modifying) therapies must be evaluated at the DMSRU annually. ${ }^{34}$ Patients attending the DMSRU consent to their clinical data being linked to administrative data for research purposes. As of 
December 31, 2010, the DMSRU database had captured data on 4409 people evaluated or treated for MS. Mean (standard deviation [SD]) clinical follow-up was 5.37 (6.46) years. Of these, 2751 had definite MS and a mean (SD) duration of clinical follow-up of 7.78 (6.64) years.

\section{Nova Scotia validation cohort}

As of September 2006, the DMSRU began capturing comorbidities at the first clinic visit and annually thereafter using a standardized patient self-reported questionnaire; a comorbidity was considered as present if it was ever reported. Patients were asked to report all comorbidities regardless of the date of diagnosis so that conditions that were not actively symptomatic, such as migraines in remission, would still be captured. As of 31 December, 2010, this cohort included 1923 people with definite MS who had a mean (SD) duration of clinical follow-up of 8.83 (6.60) years, and mean (SD) age of MS symptom onset of 33.2 (9.9) years (Table 2). Using their unique health care identification numbers, we linked the validation cohort with administrative data for Nova Scotia MS study population from 1 January, 1990 through 31 December, 2010, with January 1990 representing the earliest date for which we were granted administrative data access.

\section{Statistical analyses}

First, we compared the classification of comorbidity according to the administrative case definitions and diagnoses from the validation cohorts by computing, for each comorbidity, sensitivity and specificity of the administrative case definitions compared to self-report data with exact $95 \%$ confidence intervals based on the binomial distribution. Given that neither administrative data nor self-report can be considered the ideal or preferred source of comorbidity data, ${ }^{14,35}$ we also estimated the agreement between the data sources using kappa statistics ( $\kappa$ ) where neither data source was considered the reference standard. Kappa indicates the proportion of agreement beyond chance and is calculated as (observed agreement chance agreement $) \div(1$ - chance agreement). We interpreted the estimated $\kappa$ as follows: slight (0 to 0.20 ), fair (0.21 to $0.40)$, moderate (0.41 to 0.60$)$, substantial (0.61 to 0.80 ) and almost perfect agreement (0.81 to 1.0$){ }^{36}$ With the smallest validation cohort sizes of 606 at the study outset, we expected that this would be an adequate sample to detect $\mathrm{a} \kappa \geq 0.60$ if the comorbidity affected $3 \%$ or more of the cohort and the null hypothesis was $\kappa \geq 0.40$ (lowest acceptable $\kappa$ ), $\alpha=0.05$ and $\beta=0.20$.

Statistical analyses used SAS version 9.1 (SAS Institute Inc., Cary, NC, US).

\section{Results}

Table 2 shows the characteristics of the validation cohorts from Manitoba and Nova Scotia. Age and sex distributions for both cohorts were consistent with those expected for an MS population. ${ }^{26}$ The self-reported frequency of depression, anxiety, diabetes and heart disease were similar in both validation cohorts; however, hypertension and hyperlipidemia were less frequent in Manitoba than in Nova Scotia. In both provinces, the frequency of individual comorbidities was generally similar for administrative data and self-report (Table 2).

In Manitoba, the specificities of the administrative case definitions were over $93 \%$ for all conditions compared to selfreport (Table 3). The findings were similar in Nova Scotia, with the specificities for all

\section{TABLE 2}

Characteristics of the validation cohorts used to identify comorbidities in people with multiple sclerosis in Nova Scotia and Manitoba, Canada

\begin{tabular}{|c|c|c|c|c|}
\hline Characteristics & \multicolumn{2}{|c|}{ Manitoba validation cohort } & \multicolumn{2}{|c|}{ Nova Scotia validation cohort } \\
\hline Count (N) & \multicolumn{2}{|c|}{606} & \multicolumn{2}{|c|}{1923} \\
\hline Period covered by participant self-report & \multicolumn{2}{|c|}{ Lifetime to 2006} & \multicolumn{2}{|c|}{$\begin{array}{l}\text { Lifetime to start of data collection in } \\
\text { September 2006-December } 2010\end{array}$} \\
\hline $\begin{array}{l}\text { Time period for administrative data (hospital and } \\
\text { physician claims) }\end{array}$ & \multicolumn{2}{|c|}{$1984-04-01$ to $2007-03-31$} & \multicolumn{2}{|c|}{$1990-01-01$ to $2010-12-31$} \\
\hline Female, n (\%) & \multicolumn{2}{|c|}{$511(84.4)$} & \multicolumn{2}{|c|}{$1480(77.0)$} \\
\hline Comorbidity data source & Self-report & Administrative & Self-report & Administrative \\
\hline Hypertension (\%) & 91/576 (15.8) & 89/576 (15.4) & $466(24.2)$ & $500(26.0)$ \\
\hline Hyperlipidemia (\%) & $10 / 169(5.9)$ & $12 / 169(7.1)$ & $329(17.1)$ & $257(13.3)$ \\
\hline Diabetes (\%) & $30 / 606(4.9)$ & 29/606 (4.8) & $127(6.6)$ & $151(7.8)$ \\
\hline Heart Disease (\%) & $16 / 598(2.5)$ & $26 / 598(4.4)$ & $65(3.4)$ & $105(5.5)$ \\
\hline Anxiety $(\%)$ & $58 / 405(14.3)$ & $41 / 405(10.1)$ & 339 (17.6) & $614(31.9)$ \\
\hline
\end{tabular}

Abbreviation: SD, standard deviation.

- : Data not available. 
TABLE 3

Performance of administrative claims comorbidity definitions compared to self-report for people with multiple sclerosis in Nova Scotia and Manitoba, Canada

\begin{tabular}{|c|c|c|c|c|c|c|}
\hline Comorbidity & $\begin{array}{l}\text { Province (Number of } \\
\text { diagnosis fields) }\end{array}$ & Sensitivity $(95 \% \mathrm{CI})$ & Specificity $(95 \% \mathrm{CI})$ & PPV $(95 \% \mathrm{CI})$ & NPV $(95 \% \mathrm{CI})$ & Карра $(95 \% \mathrm{CI})$ \\
\hline \multirow[t]{3}{*}{ Hypertension } & Manitoba (1) & $0.80(0.71-0.88)$ & $0.96(0.94-0.98)$ & $0.79(0.69-0.87)$ & $0.96(0.94-0.98)$ & $0.76(0.69-0.84)$ \\
\hline & Nova Scotia (1) & $0.79(0.75-0.83)$ & $0.91(0.89-0.92)$ & $0.74(0.70-0.77)$ & $0.93(0.92-0.94)$ & $0.68(0.64-0.72)$ \\
\hline & Nova Scotia (3) & $0.79(0.75-0.83)$ & $0.90(0.89-0.92)$ & $0.73(0.68-0.76)$ & $0.93(0.92-0.94)$ & $0.68(0.64-0.71)$ \\
\hline \multirow[t]{2}{*}{ Hyperlipidemia } & Manitoba (1) & $0.50(0.21-0.97)$ & $0.97(0.94-0.99)$ & $0.60(0.26-0.88)$ & $0.96(0.92-0.99)$ & $0.51(0.25-0.78)$ \\
\hline & Nova Scotia (1) & $0.53(0.48-0.59)$ & $0.95(0.94-0.96)$ & $0.68(0.62-0.74)$ & $0.91(0.89-0.92)$ & $0.53(0.47-0.58)$ \\
\hline \multirow[t]{3}{*}{ Diabetes } & Manitoba (1) & $0.67(0.47-0.83)$ & $0.98(0.97-0.99)$ & $0.60(0.36-0.81)$ & $0.99(0.98-0.99)$ & $0.66(0.52-0.80)$ \\
\hline & Nova Scotia (1) & $0.80(0.71-0.86)$ & $0.97(0.96-0.98)$ & $0.67(0.59-0.74)$ & $0.99(0.98-0.99)$ & $0.70(0.64-0.77)$ \\
\hline & Nova Scotia (3) & $0.80(0.71-0.86)$ & $0.97(0.96-0.98)$ & $0.67(0.58-0.74)$ & $0.99(0.98-0.99)$ & $0.70(0.64-0.77)$ \\
\hline \multirow[t]{2}{*}{ Heart disease } & Manitoba (1) & $0.42(0.23-0.63)$ & $0.99(0.98-0.99)$ & $0.69(0.41-0.89)$ & $0.97(0.96-0.98)$ & $0.51(0.32-0.69)$ \\
\hline & Nova Scotia (1) & $0.58(0.46-0.71)$ & $0.96(0.95-0.97)$ & $0.36(0.27-0.46)$ & $0.99(0.98-0.99)$ & $0.42(0.33-0.52)$ \\
\hline \multirow[t]{2}{*}{ Epilepsy $^{\mathrm{a}}$} & Nova Scotia (1) & $0.47(0.34-0.61)$ & $0.99(0.98-0.99)$ & $0.51(0.37-0.65)$ & $0.98(0.98-0.99)$ & $0.48(0.36-0.60)$ \\
\hline & Nova Scotia (3) & $0.47(0.34-0.61)$ & $0.99(0.98-0.99)$ & $0.51(0.37-0.65)$ & $0.98(0.98-0.99)$ & $0.48(0.36-0.60)$ \\
\hline \multirow[t]{3}{*}{ Depression } & Manitoba (1) & $0.55(0.36-0.73)$ & $0.94(0.87-0.98)$ & $0.74(0.52-0.90)$ & $0.87(0.79-0.92)$ & $0.53(0.36-0.71)$ \\
\hline & Nova Scotia (1) & $0.49(0.45-0.54)$ & $0.82(0.80-0.84)$ & $0.53(0.48-0.57)$ & $0.80(0.77-0.82)$ & $0.32(0.27-0.36)$ \\
\hline & Nova Scotia (3) & $0.49(0.45-0.54)$ & $0.82(0.80-0.84)$ & $0.53(0.48-0.57)$ & $0.80(0.77-0.82)$ & $0.32(0.27-0.36)$ \\
\hline \multirow[t]{3}{*}{ Anxiety } & Manitoba (1) & $0.29(0.18-0.43)$ & $0.93(0.90-0.95)$ & $0.41(0.26-0.58)$ & $0.89(0.85-0.92)$ & $0.26(0.13-0.38)$ \\
\hline & Nova Scotia (1) & $0.62(0.56-0.67)$ & $0.74(0.72-0.77)$ & $0.34(0.30-0.38)$ & $0.90(0.88-0.92)$ & $0.27(0.23-0.32)$ \\
\hline & Nova Scotia (3) & $0.62(0.57-0.67)$ & $0.74(0.72-0.76)$ & $0.34(0.30-0.38)$ & $0.90(0.88-0.92)$ & $0.27(0.23-0.32)$ \\
\hline
\end{tabular}

Abbreviations: $\mathrm{Cl}$, confidence interval; NPV, negative predictive value; PPV, positive predictive value.

a Insufficient number of cases reported in Manitoba $(<5)$.

case definitions exceeding $91 \%$, except for depression (82\%) and anxiety (74\%). In both provinces, the sensitivities of the administrative case definitions were more variable than the specificities. The sensitivity was particularly low for anxiety, being as low as 29\% in Manitoba.

In Manitoba, agreement between the administrative case definitions and selfreport was substantial for hypertension and diabetes; moderate for hyperlipidemia, heart disease and depression; and fair for anxiety. Agreement was slightly lower in Nova Scotia for hypertension and hyperlipidemia but the same for diabetes and heart disease. Agreement was lower in Nova Scotia and more discrepant for depression. The specificity of the case definition for anxiety was lower in Nova Scotia than in Manitoba. Two conditions were tested solely in Nova Scotia; agreement was substantial for inflammatory bowel disease and moderate for epilepsy.

As a sensitivity analysis, we also evaluated the impact of using all 3 diagnoses submitted on the physician claims rather than the first diagnosis, but this had no meaningful impact on the performance of our case definitions (see Table 3).

\section{Discussion}

We tested the performance of administrative case definitions for common comorbidities in MS populations from 2 Canadian provinces, Nova Scotia and Manitoba. The prevalence of these comorbidities differed somewhat across populations, consistent with variation seen in other studies. ${ }^{11}$ Our study demonstrates a good overall performance for several important comorbidity definitions using health administrative data in the MS populations in these 2 provinces. This represents a major step forward in developing a pan-Canadian monitoring system for comorbidity in MS, an increasingly important issue for the population, health system and policy makers.

However, our study also highlights the importance of developing a robust methodology and fully understanding the strengths and limitations of the available data sources before moving forward with such a system. For instance, agreement between administrative case definitions differed by comorbidity.

Our findings also highlight the general importance of testing the performance of case definitions developed in one jurisdiction or population before applying them elsewhere. 
Administrative data are accessible, costeffective and population-based. However, since these data are collected for health system management they must be validated for use in research. The utility of these data may vary across populations and conditions $^{25,29}$ and validity may appear to vary depending on study design. $^{29}$ While the sensitivity of our administrative case definitions varied, specificity was high, typically exceeding $90 \%$ for physical comorbidities, consistent with our previous observations in Manitoba. ${ }^{17-19,23}$ The consistency of the performance of the case definitions in MS populations in Manitoba and Nova Scotia supports their use in other Canadian provinces. While the specificities of physical comorbidities were high, those for the mental comorbidities were somewhat lower, ranging from $74 \%$ to $94 \%$. Other validation studies of administrative case definitions in Canada also noted difficulties in distinguishing depression from anxiety when using 3-digit ICD codes due to the lack of specificity at this level. ${ }^{37,38}$ For example, the same code (300) describes dysthymic disorder (300.4), a form of chronic depression, and anxiety states (300.0). Similarly, the code 296 describes major depressive disorder single episode (296.2) and bipolar disorder single manic episode (296.0). In addition to difficulties distinguishing depression and anxiety, other efforts to validate case definitions for depression have reported poor concordance with depression as measured using the Composite International Diagnostic Interview. ${ }^{38}$ One study among people treated with antidepressants in Saskatchewan, Canada found that agreement between depression identified using physician claims and in medical records was moderate $(\kappa=0.54)$, better than we observed. ${ }^{39}$ In Manitoba, where populationbased prescription claims data are available, we had found that the best-performing administrative case definitions for psychiatric comorbidities, including depression and anxiety, used prescription claims. ${ }^{18}$ However, Nova Scotia has no populationbased prescription database. This is also the case in several other Canadian jurisdictions, thereby limiting the use of prescription claims-based definitions at the national level. Agreement between the case definitions and self-report for depression was also lower in Nova Scotia than that in Manitoba. This suggests that additional caution is required in using case definitions for psychiatric comorbidity.

Several of the case definitions we used had been originally developed for use in the general population but were considered to perform adequately in the MS population compared to other potential case definitions tested in Manitoba. ${ }^{17,19,28}$ These included the case definitions for hypertension, heart disease and inflammatory bowel disease. We used the CCDSS definition of hypertension; this definition performed similarly in the 2 MS populations studied. We found a sensitivity of $79 \%$ to $80 \%$, specificity of $91 \%$ to $96 \%$ and $\kappa$ of 0.68 to 0.76 . Studies in the general population from Ontario and Manitoba that tested these case definitions found similar sensitivities of $69 \%$ to $73 \%$, specificities of $95 \%$ and $\kappa$ of 0.67 to $0.70 .^{25,40}$ The case definition for heart disease showed moderate agreement with self-report in Manitoba ( $\kappa=0.51$ ) and Nova Scotia $(\kappa=0.42)$, consistent with findings in the Manitoba general population $(\kappa=0.55) .{ }^{25}$ For inflammatory bowel disease we applied a case definition developed in the Manitoba general population to the Nova Scotia MS population and found a sensitivity of $59 \%$, specificity of $100 \%$ and $\kappa$ of 0.71 . The specificity and agreement compares favourably to the initial findings in Manitoba where the specificity of using self-report was $91 \%$ and $\kappa$ was 0.79 to 0.80 . The sensitivity, however, was lower than that reported for the general population in either Manitoba ( $>87 \%$ ) or Quebec (97\%). The lower sensitivity of general population case definitions when applied to the MS population is also evident for diabetes and epilepsy. We had previously evaluated the performance of the CCDSS definition of diabetes in the Manitoba MS population and found a sensitivity of only $50 \%$, substantially lower than the $79.5 \%$ to $86 \%$ reported in the general population. ${ }^{25,41}$ We found that modifying the CCDSS definition to use 5 years of data rather than 2 improved sensitivity; ${ }^{17}$ this modified case definition had an $80 \%$ sensitivity in the Nova Scotia population while retaining a high specificity of $97 \%$.
For epilepsy, we found a sensitivity of $47 \%$ and specificity of $99 \%$ as compared to self-report. The definition that we used for epilepsy-1 hospitalization or 2 physician claims in 3 years-has not been tested in the general population in Canada. However, in the Alberta general population a case definition of 1 hospitalization or 2 physician claims in 2 years had a substantially higher sensitivity of $88.9 \%$ with a similar specificity of $92.4 \%{ }^{42}$ Overall, these findings suggest that case definitions developed in the general population may not perform as well when applied to specific chronic disease populations and that their validity should be assessed before they are used.

The lower sensitivity of case definitions for comorbidity in the MS population than that reported for the general population may reflect the known limitations of administrative data: comorbidity may be under-reported in hospital claims due to coding biases. ${ }^{43}$ Sensitivity of case definitions may also be influenced by the number of diagnoses coded, particularly in physician claims as much of the care for chronic disease is delivered in the outpatient setting. The number of diagnosis fields recorded on physician claims varies, being limited to only 1 in most provinces but up to 3 in British Columbia, 3 in Alberta, 3 in Nova Scotia since 1997, 2 in the Yukon before 2006 and unlimited thereafter and 11 in Nunavut. ${ }^{21}$ Such differences in coding could lead to apparent differences in disease prevalence across provinces. For this reason we evaluated the impact of using 3 diagnoses rather than just 1. Although we did not find any major influence of the number of diagnosis fields used on the sensitivity or specificity of our case definitions, these findings should be verified in those jurisdictions where 3 diagnosis fields are available.

We did not attempt to evaluate the impact of the changes in fee-for-service billing as physicians switch to alternative payment plans but that factor may also vary across provinces $^{21}$ and influence the sensitivity of administrative data.

We used self-report data as our reference standard, recognizing that no data source 
can be considered a true gold standard for comorbidity and that some of the discordance between the self-report and administrative data that we observed may be due to limitations in both data sources. Gathering robust self-report data poses its own challenges as respondents may fail to recall all medical conditions with which they have been diagnosed, may fail to report some conditions due to social desirability bias or may misunderstand diagnoses reported to them. Nonetheless, obtaining these data is often easier and more cost effective than gaining access to comprehensive medical records information. Review of the records of a single provider has been found to have lower sensitivity for chronic disease than review of the records of all providers visited by a person, ${ }^{44}$ yet it can be difficult to obtain access to all such records due to cost, privacy and other practical considerations. A comorbidity index based on administrative data outperformed a comorbidity index based on data from a single day review of hospital charts. ${ }^{45}$ While the validity of selfreport has been shown to vary by condition, such data appear to be reasonably accurate for well defined, chronic disorders that require ongoing care or that cause disability, such as those we investigated in our study. ${ }^{14}$ Further, self-report may predict health-related quality of life and functional status more accurately than medical records data. ${ }^{46}$ Together such findings suggest that, for at least some chronic conditions, self-report data are an adequate comparator for the validation of administrative case definitions.

Strengths of our study include the large cohorts involved and the availability of comparative data from Manitoba and Nova Scotia. Our findings that the performance characteristics of administrative case definitions for several comorbidities commonly observed in MS are relatively stable across jurisdictions suggests that they can be used effectively for populationbased studies investigating the epidemiology and impact of comorbidity in MS. Case definitions for psychiatric comorbidities require further optimization, and further work is needed to develop methods to assess other comorbidities of potential relevance to people with MS.

\section{Conflicts of interest and acknowledgements}

This study was supported (in part) by Don Paty Career Development grants to Ruth Ann Marrie from the Multiple Sclerosis Society of Canada, the CIHR and the Rx \& D Health Research Foundation. The funding sources had no role in the study design, collection, analysis or interpretation of the data or in the decision to submit the article for publication. The results and conclusions presented are those of the authors. No official endorsement by Manitoba Health or the Population Health Research Unit (Dalhousie University) are intended or should be inferred.

Ruth Ann Marrie receives research funding from CIHR, Public Health Agency of Canada, Manitoba Health Research Council, Health Sciences Centre Foundation, Multiple Sclerosis Society of Canada, Multiple Sclerosis Scientific Foundation and Rx \& D Health Research Foundation and has conducted clinical trials funded by Bayer and Sanofi-Aventis.

John Fisk is the Director of the endMS Atlantic Regional Research and Training Centre, funded by the Multiple Sclerosis Society of Canada. He receives research funding from the CIHR and has received grants, honoraria and consultation fees from AstraZeneca, Bayer, Biogen Idec Canada, Heron Evidence Development Limited, Hoffmann-La Roche, MAPI Research Trust, Novartis, Sanofi-Aventis, Serono Canada and QualityMetric Incorporated.

Karen Stadnyk has no conflicts of interest to declare.

Helen Tremlett currently receives funding from the Multiple Sclerosis Society of Canada [Don Paty Career Development Award]; US National MS Society [\#RG 4202-A-2 (PI)]; CIHR [MOP: \#190898 (PI) and MOP-93646 (PI)]; Michael Smith Foundation for Health Research (Scholar award) and the Canada Research Chair program. She has received speaker honoraria and/or travel expenses to attend conferences from the Consortium of MS Centers, US National MS Society, Swiss Multiple Sclerosis Society, the University of British Columbia Multiple Sclerosis
Research Program, Teva Pharmaceutical Industries and Bayer Pharmaceutical (honoraria declined) and ECTRIMS. Unless otherwise stated, all speaker honoraria are either donated to an MS charity or to an unrestricted grant for use by her research group.

Christina Wolfson receives research funding from the Multiple Sclerosis Society of Canada, Canadian Institutes of Health Research, Canada Foundation for Innovation, the National Multiple Sclerosis Society and the Public Health Agency of Canada.

Sharon Warren receives research funding from the CIHR, the Canadian Health Services Research Foundation, Alberta Health Services and SSHRC.

Virender Bhan receives research funding from the CIHR and has acted as advisor/ consultant for Bayer, Biogen Idec, EMD Serono, Novartis and Teva Pharmaceutical Industries.

Nancy Yu receives research support from the Canadian International Development Agency, the Multiple Sclerosis Society of Canada, CIHR and Manitoba Health and Healthy Living.

\section{References}

1. Gijsen R, Hoeymans N, Schellevis FG, Ruwaard D, Satariano WA, van den Bos GA. Causes and consequences of comorbidity: a review. J Clin Epidemiol. 2001; 54(7):661-74.

2. Mielke MM, Rosenberg PB, Tschanz J, et al. Vascular factors predict rate of progression in Alzheimer disease. Neurology. 2007; 69(19):1850-8.

3. Dean G. How many people in the world have multiple sclerosis? Neuroepidemiology. 1994;13(1-2):1-7.

4. Evans C, Beland S, Kulaga S, et al. Incidence and prevalence of multiple sclerosis in the Americas: a systematic review. Neuroepidemiology. 2013;40(3):195-210.

5. Warren SA, Svenson LW, Warren KG. Contribution of incidence to increasing prevalence of multiple sclerosis in Alberta, Canada. Mult Scler. 2008;14(7):872-9. 
6. Beck CA, Metz LM, Svenson LW, Patten SB. Regional variation of multiple sclerosis prevalence in Canada. Mult Scler. 2005; 11(5):516-9.

7. Marrie RA, Cutter G, Tyry T. Substantial adverse association of visual and vascular comorbidities on visual disability in multiple sclerosis. Mult Scler. 2011;17(12):146471.

8. Marrie RA, Horwitz RI, Cutter G, Tyry T, Campagnolo D, Vollmer T. Comorbidity delays diagnosis and increases disability at diagnosis in MS. Neurology. 2009;72(2): 117-24.

9. Marrie RA, Rudick R, Horwitz R, et al. Vascular comorbidity is associated with more rapid disability progression in multiple sclerosis. Neurology. 2010;74(13):10417.

10. Warren SA, Turpin KV, Pohar SL, Jones CA, Warren KG. Comorbidity and healthrelated quality of life in people with multiple sclerosis. Int J MS Care. 2009;11(1):616.

11. Marrie RA, Horwitz RI. Emerging effects of comorbidities on multiple sclerosis. Lancet Neurol. 2010;9(8):820-8.

12. The burden of neurological diseases, disorders and injuries in Canada. Ottawa (ON): Canadian Institute for Health Information: 2007.

13. Lash TL, Mor V, Wieland D, Ferrucci L, Satariano W, Silliman RA. Methodology, design, and analytic techniques to address measurement of comorbid disease. J Gerontol A Biol Sci Med Sci. 2007;62(3): 281-5.

14. Okura Y, Urban LH, Mahoney DW, Jacobsen SJ, Rodeheffer RJ. Agreement between self-report questionnaires and medical record data was substantial for diabetes, hypertension, myocardial infarction and stroke but not for heart failure. J Clin Epidemiol. 2004;57(10):1096-103.

15. Patten S. Integrating data from clinical and administrative databases in pharmacoepidemiological research. Can J Clin Pharmacol. 1998;5(2):92-7.
16. National Diabetes Surveillance System. Responding to the challenge of diabetes in Canada - First Report of the National Diabetes Surveillance System (NDSS) 2003 [Internet]. Ottawa (ON): Health Canada; 2003 [cited 2006 Jan 10]. Available at: http://publications.gc.ca/collections/Collec tion/H39-4-21-2003E.pdf

17. Marrie $\mathrm{R}, \mathrm{Yu} \mathrm{B}$, Leung $\mathrm{S}$, et al. Rising prevalence of vascular comorbidities in MS: validation of administrative definitions for diabetes, hypertension, hyperlipidemia. Mult Scler. 2012;18(9):1310-9.

18. Marrie RA, Fisk JD, Yu BN, et al. Mental comorbidity and multiple sclerosis: validating administrative data to support population-based surveillance. BMC Neurol. 2013;13(1):16.

19. Marrie RA, Yu BN, Leung S, et al. The utility of administrative data for surveillance of comorbidity in multiple sclerosis: a validation study. Neuroepidemiology. 2012;40(2): 85-92.

20. Marrie RA, Yu BN, Leung $S$, et al. The incidence and prevalence of thyroid disease do not differ in the multiple sclerosis and general populations: a validation study using administrative data. Neuroepidemiology. 2012;39(2):135-42.

21. Lix LM, Walker R, Quan H, Nesdole R, Yang J, Chen G. Features of physician services databases in Canada. Chronic Dis Inj Can. 2012;32(4):186-93

22. Health Information Management Branch. Population report. Winnipeg (MB): Manitoba Health and Healthy Living; 2008 June 1.

23. Marrie RA, Yu BN, Leung $S$, et al. The incidence and prevalence of fibromyalgia are higher in multiple sclerosis than the general population: a population-based study. Mult Scler Rel Dis. 2012;1(4):162-7.

24. Marrie RA, Horwitz R, Cutter G, Tyry T, Campagnolo D, Vollmer T. Comorbidity, socioeconomic status, and multiple sclerosis. Mult Scler. 2008;14(8):1091-8

25. Lix L, Yogendran M, Shaw S, Burchill C, Metge C, Bond R. Population-based data sources for chronic disease surveillance. Chronic Dis Can. 2008;29(1):22-30.
26. Marrie RA, Yu N, Blanchard JF, Leung S, Elliott L. The rising prevalence and changing age distribution of multiple sclerosis in Manitoba. Neurology. 2010;74(6):465-71.

27. Horton M, Rudick RA, Hara-Cleaver C, Marrie RA. Validation of a self-report comorbidity questionnaire for multiple sclerosis. Neuroepidemiology. 2010;35(2): 83-90.

28. Marrie RA, Yu BN, Leung $\mathrm{S}$, et al, Prevalence and incidence of ischemic heart disease in multiple sclerosis: a populationbased validation study. Mult Scler Rel Dis. 2013;2(4):355-61.

29. Quach S, Blais C, Quan H. Administrative data have high variation in validity for recording heart failure. Can J Cardiol. 2010;26(8):306-12.

30. Population, urban and rural, by province and territory (Nova Scotia) [Internet]. Ottawa (ON): Statistics Canada; 2011. Available at: http://www.statcan.gc.ca /tables-tableaux/sum-som/101/cst01/demo62 d-eng.htm

31. Marrie R, Fisk J, Stadnyk K, et al. The incidence and prevalence of multiple sclerosis in Nova Scotia, Canada. Can J Neurol Sci. 2013;40(6):824-31.

32. Bernstein CN, Blanchard JF, Rawsthorne P, Wajda A. Epidemiology of Crohn's disease and ulcerative colitis in a central Canadian Province: a population-based study. Amer J Epidemiol. 1999;149(10):916-24.

33. Lowe AM, Roy P-O, B.-Poulin M, et al. Epidemiology of Crohn's Disease in Quebec, Canada. Inflamm Bowel Dis. 2009;15(3): 429-35.

34. Veuglers PJ, Fisk JD, Brown MG, et al. Disease progression among multiple sclerosis patients before and during a diseasemodifying drug program: a longitudinal population-based evaluation. Mult Scler. 2009;15:1286-94.

35. Klabunde $\mathrm{CN}$, Warren JL, Legler JM. Assessing comorbidity using claims data: an overview. Med Care. 2002;40(8 Suppl): IV-26-35.

36. Landis JR, Koch GG. The measurement of observer agreement for categorical data. Biometrics. 1977;33(1):159-74. 
37. Kisely S, Lin E, Gilbert C, Smith $M$, Campbell LA, Vasiliadis HM. Use of administrative data for the surveillance of mood and anxiety disorders. Aust $\mathrm{N}$ Z J Psychiatry. 2009;43(12):1118-25.

38. Martens P, Fransoo R, McKeen N, et al. Patterns of regional mental illness disorder diagnoses and service use in Manitoba: a population-based study [Internet]. Winnipeg (MB): Manitoba Centre for Health Policy; 2004 Sep [revised 2004 Nov 18; cited 2013 Jan 24]. Available at: http://mchp-appserv .cpe.umanitoba.ca/reference/mental.health .pdf

39. West SL, Richter A, Melfi CA, McNutt M, Nennstiel ME, Mauskopf JA. Assessing the Saskatchewan database for outcomes research studies of depression and its treatment. J Clin Epidemiol. 2000;53(8): 823-31.

40. Tu K, Campbell NRC, Chen Z, Cauch-Dudek KJ, McAlister FA. Accuracy of administrative databases in identifying patients with hypertension. Open Medicine. 2007;1(1): e18-26.

41. Hux JE, Ivis F, Flintoft V, Bica A. Diabetes in Ontario: determination of prevalence and incidence using a validated administrative data algorithm. Diabetes Care. 2002;25(3): 512-6.

42. Reid AY, St Germaine-Smith C, Liu M, et al. Development and validation of a case definition for epilepsy for use with administrative health data. Epilepsy Res. 2012; 102(3):173-9.

43. Elixhauser A, Steiner C, Harris DR, Coffey RM. Comorbidity measures for use with administrative data. Med Care. 1998;36(1): 8-27.

44. Wilchesky M, Tamblyn RM, Huang A. Validation of diagnostic codes within medical services claims. J Clin Epidemiol. 2004; 57(2):131-41.

45. Luthi JC, Troillet N, Eisenring MC, et al. Administrative data outperformed singleday chart review for comorbidity measure. Int J Qual Health Care. 2007;19(4):225-31.
46. Bayliss EA, Ellis JL, Steiner JF. Subjective assessments of comorbidity correlate with quality of life health outcomes: initial validation of a comorbidity assessment instrument. Health and Quality of Life Outcomes. 2005;3:51. 